\title{
Niklas Luhmann, ¿una teoría sistémica de la democracia?*
}

\author{
Niklas Luhmann, a systemic theory \\ of democracy?
}

\author{
Laura Hernández Arteaga**
}

\begin{abstract}
Resumen
El presente trabajo reconstruye la concepción de la democracia desde la perspectiva de la teoría de los sistemas sociales de Niklas Luhmann. La sociología política de Luhmann, inscrita en la teoría de los sistemas sociales autorreferentes y autopoiéticos, es un referente heurístico para abordar el fenómeno de la democracia a la luz de un enfoque teórico que trasciende las fronteras tradicionales de la Ciencia Política contemporánea; es una perspectiva, si bien sociológica, que condensa un brebaje multidisciplinario que pretende delinear elementos teóricos para explicar fenómenos de la realidad política contemporánea.
\end{abstract}

Palabras clave: Teoría de sistemas sociales, Luhmann, sistema político, democracia, comunicación política.

\begin{abstract}
The work reconstructs the conception of the democracy from the perspective of Niklas Luhmann's theory of social systems. Luhmann's political sociology, inscribed in the theory of selfreferential and autopoietic social systems, is a heuristic referent to approach the phenomenon of the democracy in the view of a theoretical approach that transcends the traditional boundaries of contemporary political science; it is a sociological perspective that condenses a multidisciplinary concoction that seeks to delineate theoretical elements to explain phenomena of the contemporary political reality.
\end{abstract}

Keywords: Theory of Social Systems, Luhmann, Political System, Democracy, Political Communications.

Recibido: 31 de octubre 2017. Aceptado: 14 de noviembre, 2017.

* Esta es una versión revisada y corregida de la ponencia "La concepción de la democracia en Luhmann. ¿Una teoría sistémica de la democracia?”, presentada por la autora en el Congreso de la Asociación Latinoamericana de Ciencia Política (ALACIP) que se llevó a cabo en Montevideo, Uruguay, del 26 al 28 de julio de 2017.

** Doctora en Ciencias Políticas y Sociales por la UNAM. Profesora de Tiempo Completo adscrita al Centro de Estudios Políticos de la Facultad de Ciencias Políticas y Sociales, UNAM. Miembro del Sistema Nacional de Investigadores, nivel I. Líneas de Investigación: teoría de sistemas sociales como un programa de investigación; transformaciones del Estado mexicano contemporáneo, y teoría política moderna. Correo electrónico: lauraha@unam.mx 


\section{Introducción}

L

a sociología política de Luhmann, inscrita en la teoría de los sistemas sociales autorreferentes y autopoiéticos, es un referente heurístico para abordar el fenómeno de la democracia a la luz de un enfoque teórico que trasciende las fronteras tradicionales de la Ciencia Política contemporánea. Es una perspectiva, si bien sociológica, que condensa en su seno un brebaje multidisciplinario que se presenta como un enfoque cuya conceptualización se constituye en una teoría para explicar fenómenos de la realidad política contemporánea.

El argumento central que guía este trabajo es dilucidar si en el contexto general de la teoría de los sistemas sociales, es posible hablar de una teoría sistémica de la democracia. A una teoría política sistémica de la democracia se le deberían aplicar los presupuestos epistemológicos y metodológicos que Niklas Luhmann atribuye a la teoría de los sistemas sociales y del sistema político de la sociedad. Al mismo tiempo que dicha teoría sirva de faro para la explicación de fenómenos propios de las formas políticas democráticas contemporáneas. Los presupuestos teórico-analíticos que se exponen a continuación estimulan la investigación politológica, mediante la formulación de hipótesis auxiliares que alienten perspectivas holistas sobre la política y, ciertamente, sobre la democracia.

\section{Los contornos de la teoría}

La teoría de los sistemas sociales, expuesta por Niklas Luhmann en Sistemas Sociales (1998), parte del presupuesto de que la teoría misma está sujeta a la reflexión de sus operaciones lógicas abstractas de manera recursiva. Se trata, por ello, de una teoría autoimplicante y autorreflexiva; es decir, se someten a evaluación sus propias afirmaciones en y desde la perspectiva de una teoría de observación científica de la sociedad.

En las teorías específicas que, a manera de eslabones, se anudan en la teoría de la sociedad de Niklas Luhmann, articuladas también de manera autológica, se hace uso de distinciones que le posibilitan observar la sociedad desde distintos niveles de agregación. La distinción directriz es la distinción sistema/entorno.

Un sistema social emerge en cuanto cualquier comunicación tiene lugar entre los individuos (Luhmann, 1982: 70), y lo hace porque la sociedad es un orden emergente de comunicación en clave de doble contingencia; es decir, la reciprocidad de las relaciones entre ego y alter son el principio de improbabilidad de las relaciones sociales. "Los términos ego y alter deben dejar 
abierta la alternativa entre sistemas psíquicos o sistemas sociales, así como la alternativa de si estos sistemas están de acuerdo o no con un procesamiento determinado del sentido" (Luhmann, 1998a: 115). En las relaciones cotidianas, estas figuras, alter y ego, se conocen como hombres, individuos o personas, que tienen en el centro de sus relaciones sociales la comunicación ${ }^{1}$ (Luhmann, 1998a: 117).

La improbabilidad de la comunicación se estabiliza y permite la emergencia de estructuras sociales; los mecanismos que hacen posible que frente al rechazo de una oferta de comunicación también haya una alternativa de aceptación, están inscritos en el concepto de medios de comunicación simbólicamente generalizados que Luhmann retoma de Talcott Parsons. Estos medios, al estar insertos en la problematización de la complejidad y la doble contingencia, posibilitan que un sistema pueda adoptar también otros estados, sintonizando sus propios estados a la contingencia de otros sistemas (Luhmann, 1998a: 102), y constituyen un elemento central para la constitución de los sistemas de la sociedad. ${ }^{2}$

Los individuos participan de esta mediación simbólica a través de dos posibilidades de atribución: como vivencia o como acción, y es válido tanto para ego como para alter. El primero lo hace como emisor y, el segundo, como receptor. ${ }^{3}$ De ello resultan una serie de combinaciones que guían la diferenciación externa de medios-códigos simbólicamente generalizados en direcciones muy distintas. Las cuatro constelaciones de atribución se com-

${ }^{1}$ La comunicación se da sí y sólo sí hay entendimiento entre ego y alter, por eso Luhmann se pregunta: "¿hasta dónde los interlocutores deben ser capaces de entenderse para poder comunicarse?" (Luhmann, 1998: 117).

2 "La función general de los medios generalizados de comunicación -hacer transmisible la complejidad reducida y cuidar de la selectividad combinada también en situaciones altamente contingentes- es uno de los presupuestos fundamentales para la constitución de complejos sistemas de sociedad. Sin ella, la contingencia de las vivencias y del actuar no experimentaría un incremento digno de mención. Los partícipes en el sistema seleccionarían separadamente, con lo que no estaría garantizado que unos pudieran tomar como premisas de sus propias conductas las selecciones de otros. Sólo bajo estos dos presupuestos de una alta contingencia de las selecciones y de una suficiente no-arbitrariedad en las relaciones entre ellas pueden surgir sistemas complejos, capaces de dejar estructuralmente abierto y pese a ello sincronizar el cómo se comportará uno en lo concreto" (Luhmann, 1998a: 106).

3 "La diferenciación de los medios incorpora un recurso binario consistente en poder pensar dos posibilidades de atribución: interna y externa. Puesto que la comunicación sólo puede observarse cuando se distingue entre información y darla a conocer, puede ponerse el acento de la atribución o en la información (vivencia) o en el darla a conocer (acción). Y eso es válido para los dos lados: para aquel que inicia la comunicación y para aquel que en consecuencia debe decidir sobre la aceptación o el rechazo de la comunicación. Si la selección se atribuye (no importa por quién) al sistema, entonces estamos hablando de acción; si al entorno, entonces de vivencia. De manera correspondiente se distinguen entre sí los medios de comunicación simbólicamente generalizados dependiendo de si ambas posiciones sociales (ego y alter) se asumen o desde la vivencia o desde la acción" (Luhmann, 2007: 260-261). 
binan de la manera siguiente: vivencia de alter y vivencia de ego: verdad; valores; actuar de alter y vivencia de ego: propiedad/dinero; arte. Mientras que la relación vivencia de alter y actuar de ego, amor. Por último, actuar de alter y actuar de ego: poder/derecho.

Estos medios de comunicación requieren, para su funcionamiento, un código unitario que consiste en dos valores propios de un lado interior, y uno exterior; se presentan como disyunciones, como una forma de dos lados, con un valor positivo y un valor negativo, lo cual permite una facilidad de cruces de uno al otro lado, sin que el valor de partida cambie, "el código mismo se convierte en estructura invariable" (Luhmann, 2007: 281, 282).

Los códigos se nos presentan como estructuras que asumen la función de ser una regla de duplicación. Mediante ellos, los sistemas logran insertarse en la dinámica de la complejidad social. La duplicidad del sí/no del lenguaje que coadyuva al incremento de la contingencia exige "dispositivos suplementarios que, en forma de códigos simbólicos adicionales, gobiernen la transmisión efectiva de complejidad reducida" (Luhmann, 1998a: 104).

Con estas consideraciones se entiende que el poder sea el medio de comunicación del sistema político de la sociedad. Aunado a esta distinción teórica, Luhmann plantea el concepto de diferenciación ${ }^{4}$ de la sociedad, que no es otra cosa que la diferenciación del sistema social en una variedad de subsistemas. La sociedad moderna es una sociedad diferenciada por funciones $^{5}$. Cada subsistema de la sociedad (política, economía, derecho, ciencia, arte, moral, familia, afectos, etcétera) atiende su comunicación específica de la sociedad, así como problemas sociales cuya función corresponde o a la economía, o al Derecho, o a la política. La relación que se establece entre esos diversos sistemas en el entorno interno de la sociedad se produce a través de problemas de referencia; por ejemplo, el sistema político atiende el medio específico del poder (Luhmann, 2007: 536). El sistema político de la sociedad está destinado a resolver problemas sociales específicos relacionados con la toma de decisiones colectivamente vinculantes. De esta manera, desde la perspectiva teórica de los sistemas sociales, la política no se constituye en el centro que dirige y "controla" a la sociedad. ${ }^{6}$

${ }^{4}$ La "diferenciación del sistema es concebida, entonces, como la reproducción, al interior del sistema, de la diferencia primaria sistema/entorno". Es una forma reflexiva y recursiva de construcción de un sistema (Luhmann, 2007: 473). Con ello se trazan los límites entre el sistema y el entorno, mismos que suponen la existencia de otros sistemas que también se diferencian de sus entornos particulares.

${ }^{5}$ La función es un esquema lógico regulador que permite organizar un ámbito de comparación de efectos equivalentes (Luhmann, 1973: 20).

${ }^{6}$ Klaus von Beyme ha señalado que esta teoría "evita albergar expectativas exageradas respecto a la capacidad de control de la política, que han precipitado a crisis de frustración cada vez mayores a generaciones de teóricos políticos, al final de los cuales se encontraba un nuevo nivel de modestia en relación a las pretensiones de control" (Von Beyme, 1994: 223). 


\section{El sistema político de la sociedad}

Como se colige de los presupuestos teóricos expuestos, es una teoría que analiza las relaciones políticas y de poder en el marco de un subsistema autónomo de la sociedad. La autonomía no implica considerarlo de manera independiente y separada de la sociedad, antes bien hay una relación dinámica entre los distintos sistemas. Esta diferenciación y autonomía se configuran históricamente, emergiendo órdenes sociales con un gradiente de complejidad mayor que exige, de frente a la especificación funcional, una diferenciación y especialización de los propios sistemas en sus fronteras interiores.

Ciertamente, esta forma de diferenciación supone el tránsito de formas jerárquicas en el ejercicio del poder con sistemas políticos descentralizados que utilizaban fórmulas de legitimación de carácter moral y religioso. Reflexivamente, la teoría política dio cuenta de esta situación con la exposición de la justificación del poder mediante el derecho divino de los reyes, por un lado, y, por el otro, de los límites del poder cuando el Parlamento se convierte en el espacio de representación de la "sociedad" en la toma de decisiones; emerge, así, el principio de la soberanía del pueblo. Luhmann considera que si bien este tipo de soberanía es expresión de una paradoja-el pueblo se gobierna a sí mismo-, coloca en el centro a la democracia como la máxima expresión de la diferenciación funcional de la sociedad moderna, la cual "funcionará como una retroalimentación negativa sobre la operación del poder político que tiene una tendencia natural a expandirse de manera amenazadora" (Zolo, 1994: 86).

Al respecto, resulta interesante mirar la dinámica autorreferencial de los sistemas del Derecho y de la política. Teóricamente, el sistema del Derecho, para realizar sus funciones y desarrollar su código especializado, requiere también del despliegue autorreferencial del sistema político, el papel que la Constitución tiene en la contención y límites al poder es un ejemplo de ello (Luhmann, 1987: 146). La interrelación de estos dos subsistemas se da por el hecho de que ambos controlan el ejercicio de la fuerza física, reservada en último momento para ser ejercida por el Estado, como una organización del sistema político. Al mismo tiempo, el Derecho es incorporado por la política para la toma de decisiones, y el sistema político confía en que sus premisas de decisión política le permiten tratar un problema específico como si fuera un problema legal (Luhmann, 1987: 149). En este sentido, la política en la sociedad moderna es autónoma en la medida en que el conjunto de procesos mediante los cuales una sociedad estabiliza sus conflictos se da a través de los derechos; las contiendas políticas sólo pueden traducirse en 
conflictos sobre los derechos, los cuales constituyen las fórmulas mediante las que los temas sociales de la sociedad pueden asumir relevancia política.

Luhmann retoma la idea de la autonomía de lo político como un logro de la modernidad, formulada, en su momento, por Carl Schmitt. Empero, desde la perspectiva teórica de los sistemas sociales autorreferentes, el sociólogo alemán incorpora distintos niveles de agregación que permiten transitar de los aspectos más abstractos de la teoría del sistema político de la sociedad a otros de un carácter particular. Considerando la teoría de las formas de Spencer Brown, que el propio Luhmann incorpora, hacia afuera el sistema político es un subsistema más en el entorno interior de la sociedad; hacia adentro, representa una condensación de complejidad reducida. Es decir, históricamente, conforme la política se hace más compleja, se aleja de un ejercicio del poder relacionado directamente con el ejercicio de la fuerza, como lo expresaron los teóricos de la razón de Estado. Si bien, para Luhmann, la política no puede prescindir del esquema "amigo-enemigo", en la modernidad se desliza al ambiente interior del sistema político de la sociedad, a saber, la política. Esta última constituye la arena movediza en la que se fragua la creación del poder institucionalizado, a través de la contienda de los partidos políticos en las elecciones.

Como se puede colegir, lo político y la política están contenidos en esa forma de despliegue de comunicación política, la política está inserta en la lucha por el poder, por el acceso al gobierno; mientras que lo político está preñado de las decisiones políticas mediadas por las estructuras e instituciones establecidas como parte de las decisiones. Esta concepción tiene una línea de convergencia con la distinción que ha realizado Chantal Mouffe entre lo "político" y "la política". Para esta filósofa y politóloga de origen belga, la política se "refiere al conjunto de prácticas e instituciones cuyo objetivo es instaurar un orden, organizar la existencia humana en unas condiciones que son siempre conflictivas porque están atravesadas por 'lo político'” (Mouffe, 2010: 8). Para la autora, la democracia

supone el reconocimiento de la dimensión antagónica de lo político, razón por la cual sólo es posible protegerla y consolidarla si se admite con lucidez que la política consiste siempre en "domesticar" la hostilidad y en tratar de neutralizar el antagonismo potencial que acompaña toda construcción de identidades colectivas (Mouffe, 1999: 13-14).

El poder necesita ser simbolizado. La generalización simbólica del poder no es tanto el uso de los medios del poder, es decir, la fuerza física, como la capacidad de amenazar de manera creíble sobre su empleo (Luhmann, 
2000: 53). La policía y el ejército, por ejemplo, figuran y son visibles en los cuarteles, y no "hacen cumplir" el poder, pero previenen el desafío permanente a su simbolismo (Luhmann, 2000: 48). La generalización simbólica del poder sirve para que se haga política para la política, y se le reconozca como tal, de este modo se clausura operativamente el sistema. La identificabilidad del poder es condición para la recursividad de las operaciones del sistema. La sola secuencia de las operaciones que se reconocen una a otra como políticas permiten diferenciar al sistema del entorno social, precisamente es en ese entorno donde esta secuencia no se puede dar (Luhmann, 2000: 74-75).

Para Luhmann, el poder es la quintaesencia de la política. Eso no significa de ninguna manera que cada comunicación política deba contener una abierta o velada amenaza de aplicar el poder. Un político puede servirse también de otras formas de influencia: recurrir a su autoridad o a su habilidad retórica, o bien realizar una promesa en la prestación de un servicio, práctica común en el marco de las elecciones. La política puede utilizar su participación en la economía, lo que significa propiedades y medios de obtener dinero para condicionar políticamente los pagos; no obstante, se trata de formas de operación que solamente se observan como políticas porque conllevan el ejercicio del poder y de su institucionalización (Luhmann, 2000: 76-77).

Ciertamente, el poder es el medium necesario para la implementación de las decisiones colectivamente vinculantes, pero Luhmann señala la limitación del sistema político, es decir, el hecho de que sólo puede aplicar el poder para cuestiones y problemas que son regulados por el poder; o sea, para asuntos estrictamente de orden político. Con ello, el sistema no es capaz de controlar la función de otros sistemas en su entorno social, la relación que establece con ellos lo hace a través de acoplamientos estructurales. A manera de ejemplo, el sistema político establece una relación con el sistema del derecho mediante la Constitución, de esta forma ambos sistemas limitan sus expectativas mutuas. Mediante la Constitución se determina jurídicamente, conforme a Derecho, a quién le pertenece el poder de decidir quién puede ejercer el poder. Y ello, como se expone más adelante, es un problema de orden procedimental en el marco de la administración que incluye al gobierno.

Como todos los medios de comunicación generalizados, el poder tiene la forma de un código de preferencia. El lado positivo (= superioridad del poder) es el preferido, el negativo (= inferioridad del poder) no es deseado. El lado positivo del código expresa el empleo del poder, lo que se decide en las operaciones del sistema. La decisión política, por tanto, siempre se refiere a ambos lados del código: mando y obediencia (Luhmann, 2000: 88). Se trata de una relación contingente de la que emerge la producción de una doble contingencia en las relaciones de poder (Luhmann, 2000: 90), expuestas ampliamente en su libro Poder (1975). 
Se está aludiendo a la emergencia de los Estados como portadores centrales del principio de soberanía. Luhmann considera que hay tres paradojas mediante las cuales el sistema político auto-observa su proceso de diferenciación interna, una de las cuales es la que se refiere a la forma jerarquizada del ejercicio del poder, invisibilizada por medio del concepto de representación, la parte que está ubicada en el lado exterior de la forma se deja representar por la clase superior gobernante, el concepto de representación sirve para fundamentar el dominio "traído a la forma de Derecho, la semántica de la representación sobrevive al orden estratificado" (Torres, 2004: 329).

La segunda paradoja corresponde a las formas iniciales de diferenciación, con la existencia de sistemas políticos centralizados y con formas jerárquicas de organización, la paradoja de la limitación de lo arbitrario fue invisibilizada por el concepto clásico de soberanía. "El principio de soberanía conduce superficialmente la mirada sobre la unificación y diferencia de las autoridades territoriales" (Luhmann, 2000: 340). Se trata, entonces, de un sistema político que se orienta por el Estado, "soberanía ahora significa poder de un Estado [para] aplicar la fuerza pública" (Torres, 2004: 334-335).

La tercera paradoja se refiere a la democracia que toca la "estructura misma de la dominación: que cada uno, al mismo tiempo, se dé órdenes y las obedezca" (Torres, 2004: 345). Es decir, la paradoja de la democracia implica que el pueblo se gobierna a sí mismo; la expresión más clara de este momento lo constituye la idea de la voluntad general expuesta por Rousseau en El contrato social.

La última de las paradojas es una expresión de cómo la democracia es reflexionada por la teoría política, sobre una problematización, desde el ámbito de la teoría política, que da cuenta de la búsqueda de respuestas al problema central de cómo limitar el poder político; en esta reflexividad se introdujeron figuras como la Constitución, la división de poderes, los elecciones, el derecho al voto y la temporalidad de los cargos (Luhmann, 1997a: 36).

El primer código es el establecido en torno a la diferenciación entre gobernantes/gobernados, esto es, entre los que participan directamente en el gobierno y los que están excluidos de este ámbito de ejercicio. Entonces, una precondición para la constitución autónoma y diferenciada del sistema político es su capacidad para identificar a quienes detentan el poder y a quienes están sujetos al mismo. A partir de esta diferencia, los gobernados constituyen el entorno interno del sistema con el cual éste establece sus comunicaciones (Luhmann, 2000a: 76). Como se puede colegir, está al centro la distinción tridimensional que Luhmann discierne en el interior del sistema político de la sociedad.

Posteriormente, emerge una nueva diferenciación del código del poder que oscila entre los polos de la relación política gobierno/oposición. Es el código 
de la democracia, cuya paradoja afecta la estructura del ejercicio jerárquico y centralizado del poder, de tal manera que la democracia significa que el pueblo gobierna sobre el mismo pueblo (Luhmann, 2000: 353), el código binario de esta forma política es gobierno/oposición.

El código gobierno/oposición deriva del desarrollo que el código izquierda/derecha tuvo en las elecciones políticas. En las elecciones, el partido de la oposición se encuentra fuera del gobierno y está en situación para asumir el control del gobierno en una futura elección. Contrario a lo que pudiera pensarse por una lectura apresurada, el código de la democracia no invisibiliza los conflictos políticos, sino que los reconduce en el contexto de mecanismos estructurales, institucionales y de roles políticos que se van complejizando con el tiempo y conforme el sistema deviene cada vez más diferenciado en su interior.

Como ya se mencionó, la simbolización del poder y su consecuente estabilización en estructuras e instituciones, se puede observar a través de cargos públicos y se incorpora el papel del gobierno y el de la administración en el sistema político. De esta manera, se remite, estructuralmente, a un esquema en el que los cargos estatales más altos, tanto en el gobierno como en el parlamento, son ocupados por aquellos que resulten electos.

En un orden así, agrupaciones capaces de gobernar de forma duradera pero que actualmente no gobiernan, estarán listas para brindar una alternativa a la gestión pública actual. De ahí que la diferencia se conserve de forma duradera en el plano semántico, bien formalmente, con la oposición entre izquierda y derecha, bien materialmente con la distinción entre tendencias conservadoras y progresistas (Luhmann, 2016: 42).

Considerando lo expuesto, la democracia es expresión de la conformación de un sistema político complejo y de cómo es capaz de institucionalizar sus mecanismos y estructuras que le permiten hacer frente a una creciente complejidad de la sociedad moderna y de su propio entorno interior como sistema autónomo. Es decir, para la teoría de los sistemas sociales, la democracia es un término que designa la emergencia de nuevas modalidades de inclusión y de toma de decisiones colectivamente vinculantes, donde el pueblo participa de las decisiones mediante el derecho al voto. Perdura la paradoja de la soberanía, la relación con la estructura del poder político, sólo que ahora gobierna el pueblo a través de sus representantes (Luhmann, 2000: 96).

El código binario gobierno/oposición opera siempre simultáneamente en todas las decisiones políticas (Luhmann, 2000: 98). Al mismo tiempo, satisface todas las características de un código de preferencia: sólo el gobierno 
ocupa el lugar en el que se toman las decisiones colectivamente vinculantes. La oposición se lamenta, ejerce la crítica, articula demandas y generalmente refleja la contingencia de las decisiones políticas. El valor positivo "gobierno" es el valor de la designación del sistema, ambos lados de la relación figuran simultáneamente en la operación (Luhmann, 2000: 99).

\section{Evolución del código gobierno/oposición desde la distinción tradición/modernidad}

Para seguir la evolución de este código en el tiempo, Luhmann sugiere que el sistema político se aborde específicamente a partir de la distinción tradición/ modernidad. En la sociedad tradicional de la vieja Europa, de la que emergió la sociedad moderna, la oposición política no poseyó ninguna legitimidad. La dominación política se dio en un sistema de estratos sociales, fue necesario un señor para imposibilitar una disolución del orden político y desencadenarse una anarquía. Las relaciones de ejercicio del poder son propias de lo que en la filosofía política se denomina el gobierno de los hombres frente al gobierno de las leyes. El señor se expone a la rivalidad debido a la estratificación de la sociedad como al peligro de una muerte violenta; debido a ello, la dominación fue proclamada como un derecho, quienquiera que intentó oponerse al señor siempre se encontró en la posición de un rival que tuvo que presentar argumentos legales. El código legal/ilegal era el código ordenador de la política, de tal modo que toda rivalidad política estaba sujeta a esta distinción (Luhmann, 1990: 169).

A partir del momento en que aparece en la escena histórica la monarquía absoluta, en la figura central de un soberano que ocupa el escaño más alto sin subordinarse a nada ni a nadie, surge la distinción central entre gobernantes y gobernados, que es el contexto propiamente dicho del surgimiento del Estado como el portador por excelencia del poder, cuya máxima expresión es el código primario mandato/obediencia.

El cambio de una sociedad estratificada a una funcionalmente diferenciada tiene su expresión clara en el principio de inclusión de la población en los diversos sistemas de funciones. La democracia en el sistema político da cuenta de esta situación. Los cambios en la semántica de la "democracia" y de sus términos subsidiarios son un correlato de la evolución del sistema político en el contexto de la transición a la diferenciación funcional de la sociedad. Reflexivamente, el debate de los teóricos políticos de los siglos XVII y XVIII por establecer límites al poder, es expresión de este proceso de cambio. Como se puede ver, la teoría del sistema político de la sociedad 
interpela a la oposición política inscrita en un proceso de diferenciación y autonomía del sistema político de la modernidad; en la teoría política, este fenómeno se identifica en las perspectivas liberales, democráticas y hasta en el conservadurismo.

Con la transición hacia la sociedad más compleja, la distinción gobernantes/gobernados se superpone gradualmente a otra distinción que es gobierno/ oposición, tal como lo hemos mencionado. A través del código gobierno/oposición se resuelve la paradoja esencial de la democracia, mediante la cual el pueblo se gobierna a sí mismo y lo hace porque en la dimensión temporal se despliega la posibilidad de la alternancia de quienes gobiernan por los que son oposición y viceversa, en la dimensión material ocurre por la división entre los electores y los elegidos. De esta manera, en la sociedad moderna, la democracia se posiciona como la forma de organización política preponderante del sistema político, y se muestra teóricamente como "la escisión de la cima: la escisión de la cima del sistema político diferenciado mediante la distinción entre gobierno y oposición" (Luhmann, 1997a: 162-163).

\section{Política, decisión y tiempo}

En la teoría que nos ocupa, las decisiones son un elemento central del sistema político, la comunicación política se lleva a cabo mediante las decisiones; implican la selección entre varias posibilidades y dan constancia de su carácter contingente, que está abierto a opciones de futuro, por lo cual hay una relación entre política y tiempo. El que decide debe construir sus propias alternativas y habrá, seguramente, más de una posibilidad; derivado de ello, el hoy es hoy, y no es posible como mañana, y el futuro es incierto. "Lo que la decisión ofrece de manera complementaria es la construcción diferente de un futuro y de un pasado: ve posibilidades no contenidas en el pasado y delimita las posibilidades del futuro"7 (Torres, 2004: 181). La decisión presupone la indeterminación, la imposibilidad de pronosticar el futuro. Precisamente, la democracia participa de la reproducción de un futuro desconocido, pues la incertidumbre es su correlato funcional. La decisión política, como se ha dicho, necesita la incertidumbre del futuro, de tal forma que nunca es concebida como la última decisión, sino que dispone de otras

${ }^{7}$ La decisión "hecha desde el presente, cree encontrar alternativas en el futuro, y al proyectar las alternativas presupone siempre un factor de desconocimiento. No es que el futuro sea totalmente opaco, sino que lo que no se sabe es cuál de las alternativas presentes será la que se impondrá en el futuro: la proyección de posibilidades acabará encegueciendo el futuro" (Torres, 2004: 181). 
decisiones que producen sorpresas y por tanto otras informaciones, de las que se sirve el sistema para su actualización permanente.

En el nivel de la administración, este fenómeno se concreta a través de la planeación. Y no queda, por supuesto, sólo a nivel de esta organización de la política, también se puede hacer extensiva a los partidos políticos. Aquí reside, entonces, la forma en que estas organizaciones construyen sus nexos con el futuro, la consistencia y fidelidad a principios y su apertura al futuro sin romper con el pasado.

Pero la relación tiempo y política no está propensa a "desvanecerse en el aire", si bien la incertidumbre del futuro es una condición indispensable de la posibilidad de decisiones, ésta se da en un ambiente institucional y organizacional de posibilidades de decisión que también permite presentar el futuro como desconocido y aun indeterminado. Ya se señaló al respecto que la política institucionaliza la incertidumbre a través del voto.

En las democracias contemporáneas donde el pueblo se gobierna a sí mismo, lo que se traduce en un ocultamiento del carácter arbitrario del ejercicio del poder, surge la pregunta sobre los regímenes autocráticos. ¿Cómo opera la decisión en los gobiernos autocráticos? Un primer acercamiento a este cuestionamiento nos orienta más a la idea de Schmitt, según la cual el Estado es el que detenta el monopolio de la decisión, al proteger a la colectividad de decisiones amenazantes o de la falta de ellas. $O$, en la propia lógica de Luhmann, si en la democracia el pueblo es el que se gobierna a sí mismo, ¿dónde se deposita la soberanía? Aquí, como se ha mencionado, emerge una paradoja, la paradoja de la soberanía que este sociólogo alemán expone en el capítulo de las autodescripciones en la Política de la Sociedad. Así, en el caso de los regímenes autocráticos, el centro de la soberanía es más visible, es el Estado -y la personificación del mismo-, mientras que en la democracia se invisibiliza con relaciones más horizontales.

En la democracia se observa con claridad no sólo la diferencia entre código y programas, como las paradojas que arrastran la relación gobernantes gobernados. Si la democracia opera mediante el principio según el cual las decisiones deben ser tomadas de forma que todos puedan participar en el proceso de decisiones, la distinción código/programa permite que el lado negativo del código, la oposición, haga uso de ofertas que en sentido estricto no son propias de la política. A través de los programas, los partidos políticos toman agendas de la economía, de la educación y de la religión, y se presentan como la opción frente al lado positivo del código (Al respecto, Molina, 2002: 32).

La institucionalización de las elecciones como el medio para operar el código gobierno/oposición, nos presenta el escenario de partidos que, a través de un programa político, reclaman su "individualidad"; unos se autodescriben 
como conservadores, otros como progresistas, e incluso toman de la economía alocuciones relativas a políticas restrictivas o expansivas. Sin embargo, uno de los riesgos de esta operación, dice Luhmann, es que los partidos orienten la contienda política más allá de los programas -e incluso por la falta de ellos-y la conviertan en una controversia moral. Al mismo tiempo, este cambio tiene bases organizacionales, presupone la diferenciación organizacional de los partidos políticos que se distinguen de las facciones parlamentarias. Los partidos políticos son "estructuras políticas de interacción" que permiten "una organización de valores" (Luhmann, 1986: 213); como organizaciones de la política sobreviven al cambio del gobierno, pueden pasar de ser gobierno a la oposición y viceversa (Luhmann, 1990: 174).

Así, con la institucionalización de las elecciones políticas, se garantiza al sistema una incertidumbre generada en el mismo sistema. Las elecciones determinan el mecanismo mediante el cual el pueblo se gobierna a sí mismo. Es decir, mediante las elecciones, el sistema, en un primado del código democrático, estabiliza la incertidumbre a través de decisiones; y con ello, la política toma al futuro como lo desconocido y lo indeterminado, ella misma se confronta con esta característica. El ejercicio del voto y la competencia política de opciones partidistas son la expresión más dinámica de este fenómeno.

En un nivel más general y abstracto, la democracia es expresión de la diferenciación de un sistema político que se basa en decisiones que él mismo ha establecido y, a su vez, crea condiciones para la posibilidad de más decisiones que los representantes electos deben tomar. Estos últimos tienen un plazo limitado de ejercicio y, consecuentemente, no se sabe quién tomará las próximas decisiones después de las elecciones (Luhmann, 2000: 104-105). Así, la institucionalización de las elecciones políticas instala en el sistema una incertidumbre autoproducida (Luhmann, 2000: 104). En este sentido, la democracia es un logro altamente improbable de la sociedad contemporánea.

La democracia depende de un cierto grado de complejidad política y, como se ha mencionado, condensa altos niveles de contingencia en la comunicación social; su operación efectiva se puede observar a través de las estructuras, instituciones y roles que son las correas de transmisión de la toma de decisiones vinculantes. Ciertamente, estas decisiones no se toman autárquicamente, pues los destinatarios del ejercicio del poder cuentan con el respaldo constitucional que no sólo limita el poder, sino que regula los procedimientos políticos a través de los cuales se toman esas decisiones; por ejemplo, las elecciones para ocupar puestos de representación y el propio ejercicio del gobierno. 


\section{La comunicación política democrática}

Mediante la diferenciación interna del sistema político de la sociedad, en política, administración y público, es posible observar detenidamente la problematización teórica que se ha expuesto en los apartados previos. Con esta distinción, Luhmann inscribe la conceptualización sobre la democracia en una teoría de la diferenciación social. No se trata exclusivamente de una fórmula de autodescripción de la sociedad, es un logro evolutivo de la misma, es un "logro cargado de presupuestos, evolutivamente improbable, pero una auténtica conquista política" (Luhmann, 1997: 168). La democracia atiende una comunicación de la política moderna y se refiere al problema específico relativo a la relación entre gobernantes y gobernados, donde el "pueblo" es autor y destinatario del ejercicio del poder. La democracia presta al sistema político sólo su impresión específica como sistema político democrático. Como se puede observar, Luhmann toma distancia de las perspectivas clásicas de la democracia que la conciben como el gobierno del pueblo y para el pueblo. Con ésta, en el entorno interior del sistema político, se ubican los subsistemas de la política y la administración, lo cual permite, en la elaboración luhmanniana del sistema político de la sociedad, colocar problemas derivados de la democracia en el ámbito de la política; paradójicamente, la democracia se autodescribe como el gobierno del pueblo, como una especie de autoridad superior que da la impresión de fusionar la voluntad individual y la voluntad común (Luhmann, 2000: 366, citado por Czerwicw, 2008: 44-45).

Esta diferenciación del sistema político en política, administración y público es relevante, porque ahí es donde se despliega la democracia moderna. La democracia se hace posible institucionalmente porque el sistema político de la sociedad ha adquirido un gradiente de complejidad que se ha traducido en la configuración de estructuras, instituciones y roles cada vez más especializados que orientan la vida cotidiana de los órdenes políticos. Luhmann está posicionando su análisis en las democracias contemporáneas. Esta diferenciación triádica constituye un ciclo de comunicación que funciona, propiamente dicho, como un círculo formal del poder que delinea la comunicación dominante en contextos democráticos:

Cada sistema parcial está en disposición de plantear premisas de decisión para otros según el criterio de un determinado orden. Los electores y los interesados influyen en la política en diversos niveles de generalización. La política, a su vez, programa e informa a la administración igualmente en diferentes niveles de generalización. La administración vincula, mediante sus decisiones, al público en el sentido de una carga o de un beneficio. En el público, estas experiencias 
con decisiones vinculantes se convierten en motivos para el comportamiento en los otros roles como elector o como interesado (Luhmann, 2014: 121).

Como se puede colegir, para Luhmann esta diferenciación da cuenta de la evolución del sistema político en las sociedades modernas. La política surgió primero como un sistema autopoiético funcionalmente diferenciado (formulación de decisiones: Estado). Posteriormente, con el incremento de la complejidad, el sistema político tuvo que dotarse a sí mismo de nuevas técnicas de manejo de la complejidad, que lo condujeron al establecimiento de una diferenciación interna en política y administración, que permitieron canalizar diversos problemas hacia los diferentes componentes de su propia estructura interna diferenciada, reflejada en la separación de poderes. Finalmente, aparece lo público de relevancia política, que es tanto una parte del entorno del sistema político, como un componente interno del propio sistema. Luego entonces, la democracia está inserta en una compleja red de comunicaciones, estructuras, roles y procedimientos que coadyuvan al procesamiento de la complejidad de la sociedad moderna y de su sistema político.

En este círculo de comunicación, la política es concebida como el lugar de movilización del consenso, es el eslabón que posibilita la elaboración de amplios planes para la administración. En tanto, ésta es concebida como la esfera en la cual dichos planes adquieren la forma de las directrices para la concreción y ejecución de las decisiones colectivamente vinculantes, a través de políticas públicas y decisiones gubernamentales. Así, la diferenciación entre política y administración sirve como base para la construcción analítica de dos esferas de responsabilidad distintas. En este sentido, el tratamiento adecuado de las cuestiones políticas se concreta mediante una división funcional entre las decisiones de alto nivel (política) y el gobierno (administración pública) que se organizan en mediaciones institucionales cuya aceptación es generalizada (leyes, regulaciones, manuales, etcétera). Tal diferenciación de la política depende de una reconceptualización del Ejecutivo como política y de la Legislatura como administración, y de esta separación depende la eficacia y legitimidad del sistema político (King y Thornhill, 2003: 86).

Los distintos eslabones de esta cadena contribuyen de facto a la función del sistema político de la sociedad, la toma de decisiones colectivamente vinculantes, sólo que lo hacen de manera autónoma a la vez que interrelacionados, la perspectiva en la que operan cada uno de ellos es distinta.

Las condiciones de esta autonomía interna son rigurosamente diferentes para cada uno de los sistemas parciales. Se orientan según la función y estructura específicas del sistema parcial. Por consiguiente, son diferentes para la política 
y para la administración. Cabe distinguir, sin embargo, algunos rasgos generales fundamentales que son comunes a todos los sistemas parciales y que, por tanto, deben ser delineados aquí. Se encuentran en tres direcciones diferentes. Estas son la dimensión social, esto es, la cuestión acerca de con quién mantiene el sistema relaciones de interacción; la dimensión temporal, es decir, la cuestión acerca de cuándo -y en qué distribución temporal- se encuentran estas interacciones; y la dimensión objetiva, esto es, la cuestión de cuál es el sentido objetivo que llevan consigo estas interacciones entre sistema y entorno (Luhmann, 2014: 123).

La política incluye los procesos constitutivos generadores de consenso y disenso que habrán de desembocar en la toma de decisiones colectivamente vinculantes. Los partidos políticos son la arena por antonomasia de la política, tienen un papel significativo en la democracia, y constituyen un tipo singular de organizaciones que ejercen la absorción de incertidumbre en un mundo incierto (Luhmann, 2000: 271).

Ellos representan un momento de la absorción de incertidumbre. Apelan a valores a los que les hace falta todo sustrato ontológico. Son y permanecen siendo sistemas de autorganización que tan sólo pueden responder a los retos que les impone el entorno con la velocidad de sus propias estructuras -o definitivamente retrayéndose. En la medida en que ya no operan como portadores de ideología ni como donantes de puntos de identidad para su clientela, se concentran en el procesamiento general y permanente de los recursos: procesan personal. $Y$ con todo eso se hacen odiosos. Los partidos -se puede presumir-son un test de lo que una sociedad altamente diferenciada tiene que soportar de sí misma (Torres, 2004: 276-277).

La función de los partidos políticos consiste en llevar a cabo el proceso de preparación para que se lleguen a tomar decisiones que vinculen colectivamente -esto incluye también la decisión de formar parte del gobierno o seguir permaneciendo en la oposición (Torres, 2004: 273). Por el lado de la política, los partidos políticos constituyen un foro de articulación y de generalización de intereses, mediante la construcción de consensos y la transformación de preferencias en demandas a través de diferentes programas políticos.

La política prepara el terreno para que la administración tenga la legitimidad necesaria para tomar decisiones colectivamente vinculantes, para lo cual también recurre a la ley como uno de los recursos del sistema político; sólo cuando las decisiones están revestidas legalmente, las órdenes administrativas pueden reclamar responsabilidad y hacer que la política y el público, así como los destinatarios de las decisiones, estén obligados a tomarlas como 
propias. De ahí la afirmación de nuestro autor en el sentido de que cuando la política y los partidos políticos entran en relación, su sistema de referencia son los cargos que administran el ejercicio del poder; mientras que cuando el gobierno y la oposición entran en relación, su referencia es la ley. De tal manera que la administración:

Se ocupa, por el contrario, de la realización de lo políticamente posible y necesario mediante la elaboración de decisiones vinculantes, y ello según el criterio de unas premisas de decisión ya establecidas (hoy, por lo general, decididas de modo positivo). Estas dos funciones pueden rastrearse hasta en las sociedades más simples. Al menos, pueden encontrarse con el sentido de una separación entre, por una parte, la elaboración de las decisiones donde hay rivalidad acerca de la determinación del contenido y, por otra, la ejecución de las decisiones -o lo que es lo mismo, su implementación- (Luhmann, 2014: 111).

Al participar también de la comunicación política que coadyuva a la toma de decisiones colectivamente vinculantes, los procesos de decisión organizacional "programados en el Poder Legislativo, el Ejecutivo y el Judicial" (Luhmann, 2014: 131), se dan en este sistema parcial de tal manera que su función es producir decisiones vinculantes, para lo cual se establece un nexo estrecho entre política y administración. En la realidad efectiva, este proceso de interrelación implica un gran dinamismo. A través de las elecciones, otras personas llegan a los cargos de la administración; la personalidad del político tiene un significado funcional de gran utilidad, al desarrollar diferentes roles, ya sea religiosos, familiares o culturales, cuyos desempeños son secundarios para el sistema. Lo relevante para el sistema político es que los políticos tengan un mayor distanciamiento de intereses personales, pero no así de los votantes (Luhmann, 2010: 136-137).

Es interesante la formulación de lo público desde la teoría que ocupa este artículo. A diferencia de la política y la administración, considerados como sistemas internos del sistema político de la sociedad, lo público es considerado como un rol. ${ }^{8}$ Los roles del público juegan un papel importante en los procesos de estabilización de las estructuras del sistema político al proveer de consenso mediante la formación de opinión pública al sistema político, en este sentido contribuyen a la generación de legitimidad. Con ello, se permite

8 "Los roles del público no son un sistema; cada uno, por sí mismo, es un pequeño sistema que puede mantenerse relativamente constante respecto de otros roles del individuo. Esta invariabilidad se muestra, por ejemplo, en el hecho de que el individuo, tras divorciarse, sigue votando al mismo partido, que el ascenso social deja sin variación sus preferencias políticas y su influencia en la administración, o que la evolución de su propiedad no se traduce inmediatamente en actividad política (Luhmann, 2014: 288). 
que la política produzca legitimidad como recurso simbólico mediante la proposición de planes o políticas al público, o introduciendo temas públicos con cierto grado de popularidad, al debate político. Así, una vez creado el recurso de la legitimidad, es usado y preservado por la administración pública que opera mediante premisas de decisión dinámicas. "Si el ámbito de decisión de la administración queda construido con ayuda de la política de este modo, entonces la administración puede alcanzar una gran complejidad propia y, empero, puede permanecer capaz de decidir" (Luhmann, 2014: 136).

\section{La legitimidad democrática procedimental}

La sociedad moderna es para el sistema político una fuente constante de demandas que requieren respuestas, que son atendidas mediante la política democrática, algunas de las cuales incluso resultan contradictorias, en la forma de diversas políticas públicas como en programas políticos que se ofertan por los partidos políticos en los procesos electorales. Desde la administración, el sistema político debe operar en atención a esos temas y tomar decisiones que gocen de legitimidad. Luhmann considera que uno de los mecanismos de legitimidad por excelencia es el procedimiento que se relaciona con el sistema normativo, sin que ello se traduzca en señalar que la legitimación se dé en el sistema del Derecho; cabe decir que la propia positivación del Derecho y la estructura de los cargos de la administración presuponen a la política (Luhmann, 2014: 126). Esto es, la positivación del Derecho sirve de mecanismo de estabilización de las estructuras que derivan de las decisiones y, a su vez, las estabilizan. La legitimación se produce en el propio sistema político, en lo que Luhmann, en su libro Sociología política, denomina el sistema de los procesos políticos que dan cuenta del ámbito en el que se configura la creación del poder, pues "antes de que el poder legítimo pueda ser aplicado ha de ser creado" (Luhmann, 2014: 214).

Los procedimientos legitimantes, como dice Raffaele Di Giorgi, "están constituidos por las elecciones, por los debates parlamentarios y por el proceso jurídico" (Di Giorgi, 1998: 261). Estos procedimientos se constituyen en el marco regulatorio de sistemas particulares que tienen premisas de decisión distintas, en particular del sistema político, pues las premisas de decisión y las condiciones del apoyo político se condicionan mutuamente y dan lugar a una indeterminación estructural del propio sistema (Luhmann, 1980: 127).

De tal manera que la elección de los que detentan el poder está regulada a través de procedimientos jurídicos y de una legislación particular, mediante este mecanismo se produce la concreción institucional de la "voluntad 
popular" (Luhmann, 1980: 128). Es decir, los procesos políticos mediante los cuales se dilucida quién gobierna, a través de las organizaciones de partidos políticos, como son las elecciones políticas, se constituyen en un método para el reclutamiento de cargos públicos. Para tal efecto se requiere una serie de procedimientos como el tipo de sistema de elección, el registro de los electores, de los candidatos, los tiempos de elección y las reglas de asignación de cargos políticos. Estos procedimientos, como se ha mencionado, son el alma de la competencia política propiamente dicha y son regulados jurídicamente. Se trata, dice Luhmann, de una legitimación por procedimiento jurídico. Sólo una elección democrática puede legitimar la soberanía.

También aquí la nueva autonomía y diferenciación dinámica del sistema político muestra que la legitimidad no puede ser fundada simplemente en estructuras fijas de la sociedad, sino que debe ser elaborada en el mismo sistema político. Se trata de un proceso que, mediante procesos de comunicación continuos, es remitido al entorno del sistema político, a la sociedad, y que está controlado de acuerdo a ésta (Luhmann, 2014: 215-216).

A diferencia de las sociedades primitivas, el desempeño de los cargos tiene una gran movilidad. Los roles, sin embargo, se estabilizan: los personajes que detentan un cargo como presidente o diputado cambian, pero el cargo se estabiliza en una estructura del sistema. En los sistemas políticos diferenciados, los roles se individualizan, pero mantienen su carácter de estructuras estabilizadas socialmente; esto no quiere decir que el conflicto político desaparezca, sino que es mediado institucionalmente a través de la elección política, es decir, hay una especificación de un político que gobierna de frente a un elector que es parte del público.

Lo que en la teoría política es concebido como un problema de libertad e igualdad y un Derecho democrático, Luhmann lo concibe como un principio de indiferencia y de especificación de motivos. El acceso de los electores a la política es independiente de su rol en otros ámbitos sociales, como padre de familia, profesor y, ciertamente, en su papel de elector goza de la garantía del secreto. Desde la perspectiva de Luhmann, esto posibilita la intensificación de la comunicación política y una autonomía de la política que, a su vez, también eleva su dependencia de la política (Luhmann, 1980: 135). Esta circularidad conlleva una estabilización de estructuras e instituciones que son reguladas procedimentalmente por el Derecho. Para Luhmann, la diferenciación del procedimiento jurídico de las elecciones está asegurada por tres principios: a) el acceso universal al voto, $b$ ) la igualdad del valor del voto, y c) por el carácter secreto del mismo. El presupuesto de estos principios es el carácter abstracto e individualizado del elector. 
Otra función del proceso electoral es formar alternativas y mantenerlas abiertas. La institucionalización de las elecciones como un procedimiento legal es conveniente en la medida que permite dar atención al conflicto propio del poder mediante la reproducción de las oposiciones; la oferta del candidato es seleccionada por el elector que se inclinó por esa oferta, produciéndose, de esta manera, la victoria electoral de esa opción política que, a la postre, resulta provisional porque las elecciones se repiten periódicamente. Este proceso de estabilización de la elección permite mantener abierta la esperanza de los contendientes -considerados adversarios-, para que en una siguiente elección resulten ganadores (Luhmann, 1980: 135).

Es interesante mencionar que para este sociólogo, la legitimidad a través de los procesos electorales está más relacionada con las estructuras del sistema político y de la política propiamente dicha que con los individuos o personas. Para él, la diferenciación de roles en el proceso electoral es expresión de una transmisión psíquica de la toma de decisiones al elector. Se produce, de esta manera, una situación que neutraliza la influencia de las estructuras sociales sobre el sistema político; mediante esta experiencia el elector, que lo es aisladamente, está en libertad de elegir y lo hace inclinado por su preferencia política, por la propaganda de un candidato, por una inclinación familiar, pero su decisión se limita a votar por el candidato del gobierno o de la oposición. Para decirlo de manera abstracta, este aspecto experiencial de la persona que participa de las elecciones se repite periódicamente y se convierte en un mecanismo por medio del cual el sistema político se auto sanciona y la decisión político-administrativa del gobierno previo se confirma o se rechaza (Luhmann, 1980: 140-141). En las elecciones, los políticos tratan de convencer al pueblo de que voten por ellos. Para ello, se pone mucho cuidado en la presentación más ventajosa del programa político y los acentos fuertemente morales sirven para eso, para insinuar que sólo se alcanzarían determinados acuerdos y motivaciones políticas en el sentido de los buenos y justos (Luhmann, 2000: 260).

De suyo, Luhmann considera que la democracia, donde priva el código secundario gobierno/oposición, no puede concebirse sin la existencia del público que de suyo es incorporado en la toma de decisiones colectivamente vinculantes. Es por ello que en esta relación entre la administración y el público se forma el contrapoder.

La representación dominante sobre el poder parte del supuesto de que la administración al tomar decisiones es quien domina al público afectado. El problema aparece después en el control jurídico que se le impone a la forma en que las órdenes fueron realizadas: por tanto, como un problema de Estado de Derecho. El ciudadano apelará a quedar protegido por el Derecho; o si no, reaccionará en el momento de las elecciones (Torres, 2004: 268-269). 
Precisamente, en el contexto de sistemas políticos democráticos, Luhmann incorpora el concepto de opinión pública, el cual está ubicado en el lado externo de los sistemas internos de la sociedad; lo "público" es un símbolo de intransparencia que se produce cuando lo que se comunica se transparenta (Luhmann, 2000: 285).

Circunscribe su análisis en el tratamiento del sistema político de la sociedad, pues es en éste donde se decide cuando un evento, situación u opinión deviene en una comunicación política. La opinión pública es un medio ${ }^{9}$ de formación de opiniones, es un medio para la observación de segundo orden. "Es el Espíritu Santo del sistema", dice Luhmann. Se puede considerar como una apariencia autoproducida por la comunicación pública, una especie de espejo en el cual se refleja la comunicación, es decir, permite la auto-observación del sistema político. Se puede observar, asegura Luhmann, como un brillo autocreado a través de la comunicación, como una especie de espejo en el cual la comunicación se refleja a sí misma (Luhmann, 2000: 286 y 288). La opinión pública permite la inclusión de los excluidos, la presencia de los ausentes. Esta característica "hace necesario que la comunicación se escenifique" y la observación se orienta a esa escenificación, con lo cual se "desembaraza de la presión de la presencia y entonces la comunicación se desarrolla tan sólo en el nivel de comunicación entre ausentes" (Torres, 2004: 297).

A su vez, la opinión pública es el entorno interno del sistema de la políti$\mathrm{ca}$, de las organizaciones políticas y de las interacciones. En el ámbito de la política activa, los políticos son vistos como observadores de primer orden, los hombres políticos se observan y observan a los otros en función de lo que deben pensar de una acción que se expone a ser observada. En política, las elecciones políticas tienen un papel de competencia, constituyen las arenas movedizas de la opinión pública. Como se puede apreciar, la opinión pública se compone de actores que reaccionan a situaciones en tanto personas o grupos, con lo que persiguen sus propios intereses. Su acción se ve en las características personales o de grupo, en su afiliación a un partido o en sus posturas ideológicas (Luhmann, 2000: 291-292). Por ello, la opinión pública no es un medio empleado de manera exclusiva por el gobierno, también es utilizado por la oposición como el lugar para hacer valer sus programas mediante la contienda político-electoral.

${ }^{9}$ La definición del medium como la "diferencia entre el sustrato y la forma temporales del medium descansa en la simultaneidad visible de la interacción de la palabra y el silencio. Esta asimetría de roles entre palabra y silencio es asegurada por los medios técnicos. Precisamente esta forma de simultaneidad tiene un precio: el anonimato de los participantes, la abstracción de sus cualidades y de sus estados, y de hecho la imposibilidad de controlar las repercusiones de la comunicación a partir del impacto buscado" (Luhmann, 2000: 289). 
Lo que se pone en escena para un observador, se ve en la obtención del consenso. Incluso y sobre todo la cultura del conflicto propio de la democracia puede ser usado por esta expectativa. Así, desde que los conflictos son autorizados e institucionalizados en el cuadro de la oposición política, en particular en lo que concierne a los conflictos intra-organizacionales (y sobre todo interno a los partidos), también se espera que las organizaciones hablen con una sola voz (Luhmann, 2000: 294).

En contextos democráticos, la opinión pública favorece un deslizamiento de la dupla: consenso/violencia a la de consenso/disenso. Es decir, favorece el esquema de observación consenso/disenso y oculta el punto ciego de este esquema, objeto de la política: propiamente dicha la disposición de la violencia legítima organizada por el Estado (Luhmann, 2000: 294-295). En esta misma línea de argumentación, en la toma de decisiones colectivas, la democracia permite mantener el juego abierto en la construcción de consensos, sin invisibilizar el disenso. Es por ello que la opinión pública es crucial para el proceso político de la comunicación democrática. No es de extrañar que para Luhmann, la formación institucionalizada de la opinión se lleve a cabo en las democracias representativas al operar de manera independiente del ámbito de gestión de la administración, la cual obtiene la aquiescencia del elector para que decida (Luhmann, 1971: 38). La opinión pública también es el espacio de escenificación de los valores, de los juicios o de la divulgación de las faltas en el desempeño de los políticos en la administración, lo cual tiene clara resonancia en la política; razón por la que el elector se ve influenciado por la visibilización que le permite la opinión pública.

Cabe hacer un señalamiento a propósito de la relevancia que los medios de comunicación de masas tienen en las sociedades contemporáneas. Para Luhmann es falso que los medios masivos produzcan una opinión pública de la política y que tengan una influencia creciente sobre la política, pues los medios dan a conocer intereses y sirven también al entretenimiento. La opinión pública no se crea sólo en los medios masivos, que es un observador de primer orden; la opinión pública, como se ha mencionado, opera sobre el plano de la observación de segundo orden (Luhmann, 2000: 309).

\section{Conclusiones}

La concepción de la democracia en Luhmann no sigue los cánones de la teoría clásica de la democracia, que pretende establecer normas y valores que les son comunes y aplicables de manera universal. En este sentido, se trata de una perspectiva no normativa de la democracia, lo cual también im- 
plica una toma de postura, y lo hace en los términos en que la "acción política en la democracia debe desenvolverse al nivel de una mayor amoralidad" (Luhmann, 1997a: 167) al ser considerada como un logro de la sociedad. La democracia "precisa de un estilo distinto de amoralidad, a saber: la renuncia a la moralización del oponente político" (Luhmann, 1997a: 168). Ello, no obstante, no excluye que en una contienda política la oposición haga uso de programas que apelan a valores que los posicionan a la izquierda o a la derecha, o les impriman el atributo de progresista o conservador, e incluso se apele en una campaña a valores morales.

Al mismo tiempo, se posiciona en línea de continuidad con la perspectiva liberal, al considerar que la democracia sustantiva no es políticamente realizable, porque exigir "una participación intensa y comprometedora de todos, significaría ejercer violencia sobre el principio democrático", una perspectiva de este tipo tiene que "llegar a la conclusión que ella es incompatible con la racionalidad" (Luhmann, 1986: 208).

La teoría de los sistemas sociales autorreferentes y autopoiéticos en la que se inscribe el sociólogo alemán Niklas Luhmann, formula una teoría de la diferenciación funcional del sistema político de la sociedad que enriquece el debate actual sobre la democracia. Si bien el tratamiento sobre la democracia no está puesto en el mismo nivel de generalización que su teoría de la sociedad, la democracia presupone la diferenciación del sistema político en los subsistemas de política, administración y público; esta distinción se convierte en un recurso heurístico para dar cuenta de la complejidad social en la que emerge y se lleva a cabo la democracia. La democracia, como se ha expuesto en este trabajo, está inserta en un bucle de funciones, comunicaciones, códigos, estructuras, roles y procedimientos que la someten a constantes cambios, los que, en un plano más abstracto, impactan en el sistema político.

En este sentido se puede hablar de una teoría de la democracia inserta en la diferenciación funcional del sistema político, cuya reconstrucción teórica se puede hacer utilizando la propia lógica de la teoría de los sistemas sociales, expuesta magistralmente por Niklas Luhmann en Sistemas sociales, Sociedad de la sociedad y La política de la sociedad.

\section{Bibliografía}

Beyme, Klaus von (1994), Teoría política del siglo xx. De la modernidad a la posmodernidad, Madrid, Alianza Universidad.

Czerwick, Edwin (2008), Systemtheorie der Demokratie. Begriffe und Strukturen im Werk Luhmanns, Verlag für Sozialwissenschaften, vs Verlag für Sozialwissenschaften. 
De Giorgi, Raffaele (1998), Ciencia del Derecho y legitimación, México, UIA. Luhmann, Niklas (1971), "Komplexität und Demokratie", en Politische Planung Aufsätze zur Soziologie von Politik und Verwaltung, Germany, Westdeutscher Verlag Opladen, pp. 35-45.

Luhmann, Niklas (1973), Ilustración Sociológica y otros ensayos, Buenos Aires, Editorial Sur.

Luhmann, Niklas (1980), Legitímacáo pelo Procedimento, Brasília, Editora Universidade de Brasília (Colecáo Pensarnento Político, 15).

Luhmann, Niklas (1982), The Differentiation of Society, New York, Columbia University Press.

Luhmann, Niklas (1986), "Complejidad y democracia", en Marco Cupolo (comp.), Sistemas políticos. Términos conceptuales, temas del debate italiano, México, UAM Azcapotzalco, pp. 199-218.

Luhmann, Niklas (1987), "Machtkreislauf und Recht in Demokratien”, in Niklas Luhmann, Soziologische Aufklärung 4. Beiträge zur funktionalen Differenzierung der Gesellschaft, Germany, Westdeutscher Verlag, Opladen, pp. 142-151.

Luhmann, Niklas (1997), "El futuro de la democracia”, en Teoría política en el Estado de Bienestar, España, Alianza Editorial, $2^{a}$ reimpresión, pp. 161-170. Luhmann, Niklas (1998), Sistemas sociales. Lineamientos para una teoría general, Barcelona, Anthropos, Universidad Iberoamericana.

Luhmann, Niklas (1998a), "Consideraciones introductorias a una teoría de los medios de comunicación simbólicamente generalizados", en Complejidad y modernidad. De la unidad a la diferencia, Madrid, Trotta, pp. 99-130.

Luhmann, Niklas (2000), Die Politike der Gesselschaft, Francfort/Main, Suhrkamp.

Luhmann, Niklas (2007), La sociedad de la sociedad, México, Herder.

Luhmann, Niklas (2010), Politische Soziologie (André Kieserling, editor), Berlin, Suhrkamp Verlag.

Luhmann, Niklas (2014), Sociología política, Madrid, Trotta.

Luhmann, Niklas (2016), "Distinciones directrices. Sobre codificación de semánticas y sistemas", en Distinciones directrices, Madrid, Centro de Investigaciones Sociológicas, pp. 27-47.

Mouffe, Chantal (1999), El retorno de lo político. Comunidad, ciudadanía, pluralismo, democracia radical, España, Paidós.

Mouffe, Chantal (2010), "Política agonística en un mundo multipolar", CIBOD, Serie Dinámicas Interculturales, núm. 15, Barcelona, pp. 1-35.

Torres Nafarrate, Javier (2004), Luhmann: la política como sistema, México, Fondo de Cultura Económica/Universidad Nacional Autónoma de México/ Universidad Iberoamericana.

Zolo, Danilo (1994), Democracia y complejidad, Argentina, Nueva Visión. 